\title{
Correlates of Variation in Guinea Worm Burden among Infected Domestic Dogs
}

\author{
Sarah Anne J. Guagliardo, ${ }^{1,2 \star}$ Ryan Wiegand, ${ }^{1}$ Sharon L. Roy, ${ }^{1}$ Christopher A. Cleveland, ${ }^{3}$ Hubert Zirimwabagabo, ${ }^{2}$ \\ Elisabeth Chop, ${ }^{2}$ Philippe Tchindebet Ouakou, ${ }^{4}$ Ernesto Ruiz-Tiben, ${ }^{2}$ Donald R. Hopkins, ${ }^{2}$ and Adam J. Weiss ${ }^{2}$ \\ ${ }^{1}$ Parasitic Diseases Branch, Division of Parasitic Diseases and Malaria, Centers for Disease Control and Prevention, Atlanta, Georgia; ${ }^{2}$ Guinea \\ Worm Eradication Program, The Carter Center, Atlanta, Georgia; ${ }^{3}$ Southeastern Cooperative Wildlife Disease Study, University of Georgia, Athens, \\ Georgia; ${ }^{4}$ Guinea Worm Eradication Program, Ministry of Public Health, N'Djamena, Chad
}

\begin{abstract}
The Guinea Worm Eradication Program has been extraordinarily successful-in 2019, there were 53 human cases reported, down from the estimated 3.5 million in 1986. Yet the occurrence of Guinea worm in dogs is a challenge to eradication efforts, and underlying questions about transmission dynamics remain. We used routine surveillance data to run negative binomial regressions predicting worm burden among infected dogs in Chad. Of 3,371 infected dogs reported during 2015-2018, 38.5\% had multiple worms. A multivariable model showed that the number of dogs in the household was negatively associated with worm burden (adjusted incidence rate ratio $[\mathrm{AIRR}]=0.95,95 \% \mathrm{Cl}: 0.93-0.97, P<0.0001$ ) after adjusting for dog age (AIRR $=0.99,95 \% \mathrm{Cl}: 0.96-1.01, P>0.1$ ). This could relate to the amount of infective inocula (e.g., contaminated food or water) shared by multiple dogs in a household. Other significant univariable associations with worm burden included dog history of Guinea worm infection (IRR $=1.30,95 \% \mathrm{Cl}: 1.18-1.45)$ and dog owners who were hunters (IRR $=0.78,95 \% \mathrm{Cl}: 0.62-0.99, P<0.05$ ) or farmers (IRR $=0.83,95 \% \mathrm{Cl}: 0.77-0.90, P<0.0001)$. Further analysis showed that the number of dogs in the household was significantly and positively correlated with nearly all other independent variables (e.g., owner occupation: farmer, fisherman, or hunter; dog age, sex, and history of Guinea worm). The associations we identified between worm burden and dogs per household, and dogs per household and owner characteristics should be further investigated with more targeted studies.
\end{abstract}

\section{INTRODUCTION}

The global Guinea worm eradication campaign was launched in 1980, at a time when the annual number of human cases was estimated to be 3.5 million in 21 countries worldwide. ${ }^{1}$ The program has been enormously successful, reducing the number of human cases to just 54 in four countries in 2019. ${ }^{2,3}$ Yet eradication efforts are challenged by ongoing transmission of the parasite in domestic dogs in Chad, ${ }^{4,5}$ where most human and canine cases also occur today. ${ }^{2,3}$ Although canine cases of Guinea worm have been historically well documented, ${ }^{4,6-13}$ questions remain about transmission dynamics in dogs. For instance, how are dogs becoming infected, and what risk factors influence the probability of canine infection? By extension, how might we design and tailor interventions to more effectively reduce infection risk in dogs?

In both dogs and humans, infection with Dracunculus medinensis (the causative agent of Guinea worm disease) occurs upon ingestion of freshwater copepods that are infected with larvae. About 90 days postinfection in a definitive host, male and female larvae mate. Gravid female worms grow to a length of up to approximately $100 \mathrm{~cm}$ and migrate to subcutaneous tissues, usually on a lower extremity. The mature female worm emerges from the skin approximately 1014 months after initial ingestion. A blister forms and subsequently ruptures. This lesion causes an intense burning sensation, often prompting the host to seek relief by submerging the lesion in water. ${ }^{14}$ Contact with water cues the adult female worm to release first-stage larvae (L1s) and fluid from the uterus. ${ }^{15}$ First-stage larvae are then ingested by cyclopoid copepods; during the next 10-14 days in this intermediate host, the larvae undergo two molts and develop

*Address correspondence to Sarah Anne J. Guagliardo, U.S. Centers for Disease Control and Prevention, 1600 Clifton Rd NE, Atlanta, GA 30333. E-mail: sguagliardo@cdc.gov into the infective L3 stage. ${ }^{15}$ The cycle starts anew when a host ingests copepods infected with the L3 larvae.

Infected dogs in Chad occur in two main areas, Moyen Chari and Chari Baguirmi Regions, ${ }^{16}$ and peak in the month of June. ${ }^{4,16}$ Infection reoccurs in about $13 \%$ of dogs, and most infected dogs are owned by people of the Sara Kaba ethnicity. ${ }^{16}$ Previous works have reported an average of about two worms per dog, ${ }^{16}$ with a highly aggregated distribution, but correlates of elevated worm burden in dogs remain poorly understood. One analysis showed that the overall risk of infection in dogs increased with the consumption of fish and decreased with the provision of water to dogs, but no associations were identified between worm burden and the variables considered (e.g., age, sex, body condition, water provision, and proportion of fish in the diet), after accounting for extreme observations. ${ }^{17}$ From the standpoint of disease control and eradication, a host with multiple worms warrants particular attention because it has the potential to contaminate many water sources, possibly over a longer period of time when worms emerge at different times. ${ }^{14}$ In fact, on first immersion of a gravid female worm into water, she can release nearly 600,000 free-swimming L1s. ${ }^{14}$ Therefore, a host infected with multiple adult Guinea worms has the potential to contaminate water sources with hundreds of thousands, if not millions, of larvae. A photograph of a dog with multiple lesions from emerging Guinea worms is shown in Figure 1. In an effort to advance scientific understanding of $D$. medinensis transmission in domestic dogs, we used surveillance data from the Chad Guinea Worm Eradication Program (CGWEP) to explore differences between dogs with high-intensity infections (many worms) and dogs with low-intensity infections (few worms). Findings from this analysis may generate additional research questions about transmission dynamics and inform decision-making about how to prioritize surveillance and health education efforts.

\section{METHODS}

Surveillance data. In Chad, most $D$. medinensis infections in humans and in animals are located along the Chari River, ${ }^{4}$ which is approximately $1200 \mathrm{~km}$ in length from its most distant 


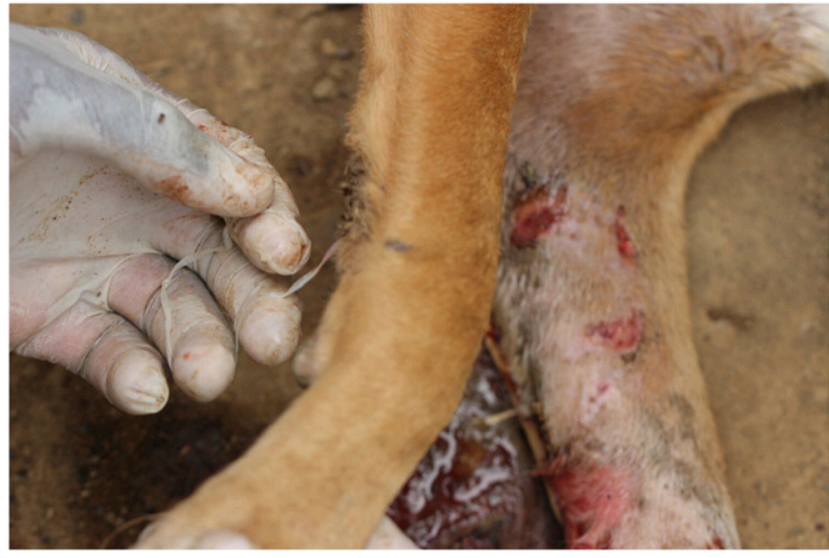

FIGURE 1. A dog with an emerging guinea worm and several lesions. Although most dogs present with a single Guinea worm, the observed range of worms per dogs is $1-79$, as detected by the CGWEP surveillance system. The dog in this photo has an emerging worm (foreground) in addition to several lesions from other worms (right). (Photo credit: Robert Hartwig, The Carter Center.) This figure appears in color at www.ajtmh.org.

point source to Lake Chad. Accordingly, most of the areas under active surveillance are also located in the same region.

The CGWEP, including its surveillance system, is managed by the Ministry of Public Health, and is described in detail elsewhere. ${ }^{16}$ In brief, surveillance is organized into three tiers of varying degrees of intensity. Villages under level 1 (proactive village-based) surveillance undergo house-to-house monitoring for cases multiple times per week where endemic transmission of Guinea worm has been documented. Level 1 villages receive the most resources, training, and supervision. Level 2 villages are in geographic proximity to level 1 surveillance and are at high risk of importation of human and/or canine infections but may not have reported human or animal cases. Level 2 areas are also under active surveillance, with less supervision than in level 1. Level 3 surveillance occurs in non-endemic areas that have not reported Guinea worm cases since 2010. This is a passive system which informs residents about Guinea worm disease and encourages them to report persons or animals with possible signs or symptoms of the infection (rumors) to local health clinics. Information about these rumors is relayed to the CGWEP system for further investigation.

When possible, Guinea worm infections are identified, and CGWEP field staff respond quickly to "contain" the case and prevent contamination of water sources, thus interrupting the transmission cycle. In the context of canine cases, "containment" of a Guinea worm is achieved when all of the following criteria are met: 1) the dog is detected within 24 hours of notification of the suspect canine case; 2) the dog has not entered any water source since the detected worm emerged; 3) the CGWEP field staff have properly managed the case, by tethering the dog before worm emergence and until all Guinea worm wounds have fully healed (to prevent contamination of water sources); and 4) the containment process, including (visual) verification that the case is Guinea worm disease, is validated by a supervisor within 7 days of the emergence of the worm. An infected dog is considered to be contained if all of the emerging Guinea worms associated with that dog meet the above criteria. For the purposes of surveillance, visual validation of the worm by a trained supervisor is sufficient to diagnose and count the case as a Guinea worm. (Surveillance data are only collected on dogs that have been confirmed as having Guinea worm by a supervisor.) Under certain circumstances, such as a new location in which Guinea worm has not been previously detected or epidemiologically important circumstances such as near a border, the worm is sent to the U.S. CDC for laboratory diagnosis as $D$. medinensis.

Statistical analysis. Descriptive analysis. We explored the distribution of worm burden in dogs by calculating the proportion of dogs that had one versus multiple worms. Increases in observed worm burden in dogs over time might point to improvements in early detection of worms. To assess this, we calculated the median number of worms per dog by calendar year for the period of interest (2015-2018). We further assessed whether successful containment varied significantly by worm emergence order among dogs with two, three, and four worms using Pearson's chi-squared test. (This was only conducted for dogs with up to four worms, as dogs with two, three, and four worms account for $83.9 \%$ of dogs with multiple worms.) To compare with previous works exploring the distribution of infected dogs irrespective of worm burden, ${ }^{16}$ the proportion of dogs with multi-worm infections were assessed in terms of their distribution over space (Region) and time (month). Graphs were produced in the $R$ package ggplot2. ${ }^{18}$

Negative binomial regression models. Negative binomial regression models were used to explore correlates of worm burden (count of worms per dog) by canine demographics and variables related to owner characteristics. Some variables were only collected during certain years-for example, whether the dog was used for hunting (only available for 2017 and 2018 data) or guarding (only available for 2018). Accordingly, we developed two different sets of models using datasets from 1) 2015 to 2018 and 2) 2018 (Supplemental Table S1). Independent variables assessed included dog age and sex, owner occupation (fishermen or hunters in comparison with other occupations), owner ethnicity (Sara Kaba versus all other groups), the number of dogs in the household (asked of the dog owner during surveillance investigation), whether the dog came from a fishing village (defined as a village in proximity to water in which the majority of inhabitants fish), whether the dog had a history of a Guinea worm infection in a different year (asked of the dog owner during surveillance investigation), and dog use (hunting, guarding, or both).

Negative binomial regression models were conducted in SAS 9.4 (Cary, NC), and all tests used a $5 \%$ level of significance. The lasso method was used for model selection. ${ }^{19}$ This approach minimizes the sum of squared errors while simultaneously limiting the sum of the absolute values of the coefficients. The Akaike information criterion value was used as the penalty factor.

For all models, an offset parameter was included to account for differences in the estimated amount of time (in months) that an individual dog was under surveillance. If a dog was initially detected in 2015, for example, that animal has a greater probability of having more worms detected by the surveillance system during 2015-2018 than a dog detected in 2018 as a result of increased amount of time under surveillance. The time under surveillance was calculated as the number of days (scaled to months) from initial detection until the last day of the year for the period of interest (December 31, 2018). The number of time under surveillance was log-transformed to 
conform to the link-log function in the negative binomial regression models. We assessed multicollinearity of all covariates using a condition index cutoff of $<15$.

We conducted a secondary analysis using data from 2015 to 2018 to assess correlations between covariates. Univariable regression models were fitted, where the outcome variables were the significant covariates from the final multivariable model described earlier.

Sensitivity analysis. As previously reported, the maximum number of worms per dog for the years 2015-2018 was 79 (observed in 2016). ${ }^{16}$ This extreme observation could influence the results of statistical modeling. We therefore ran the regression models on the full dataset (with the extreme observation) and then performed sensitivity analyses with a truncated dataset (without the extreme observation) for the years 2015-2018.

\section{RESULTS}

Descriptive statistics. Of the 3,371 infected dogs detected by the surveillance system in the years 2015-2018, 2,073 dogs (61.5\%) had just one worm and 1,298 (38.5\%) presented with multiple worms. Dogs with four worms or less represented $94 \%$ of the data. The distribution of worms per dog remained consistent for all years during the period of interest (Supplemental Table S2 and Figure S1). Successful containment varied significantly by worm emergence order (Table 1, $P<0.01$ for all tests); worms that emerged after the first worm were more likely to be contained. This finding held for dogs with two worms, dogs with three worms, and dogs with four worms. Similar to the spatiotemporal distribution of all infected dogs, ${ }^{16}$ most of the dogs with multi-worm infections were located in Moyen Chari Region (41\%), followed by Chari Baguirmi (34\%), and multi-worm infections were detected from March through August, peaking in June.

Negative binomial regression models. Results from all negative binomial regression models are summarized in Figure 2. For all datasets (2015-2018, 2015-2018 truncated, and 2018 data), dogs with a history of previous Guinea worm infection in a different year were more likely to have high worm burden. Multivariable modeling results were consistent across datasets, and we found no evidence of significant multicollinearity among covariates.

Univariable modeling results for the years 2015-2018 showed a positive correlation between dog history of Guinea worm infection and the number of worms emerged (incidence rate ratio, $\mathrm{IRR}=1.30,95 \% \mathrm{Cl}: 1.18-1.43, P<0.0001)$ (Table 2). Factors negatively associated with worm burden included the number of dogs in the household (IRR $=0.95,95 \% \mathrm{Cl}$ : 0.93-0.97, $P<0.0001)$, dog owners who were hunters (IRR = $0.78,95 \% \mathrm{Cl}: 0.62-0.99, P<0.05)$, and dog owners who were farmers (IRR $=0.83,95 \% \mathrm{Cl}: 0.77-0.90, P<0.0001)$. The final multivariable regression model showed that the number of dogs in the household was negatively associated with worm burden (adjusted incidence rate ratio $[\mathrm{AIRR}]=0.95,95 \% \mathrm{Cl}$ : $0.93-0.97, P<0.0001$ ) after adjusting for dog age (AIRR $=0.99$, 95\% Cl: 0.96-1.01, $P>0.1$ ) (Table 2). In other words, for each additional dog in the household, we observed a $5 \%$ decrease in the number of worms per dog. Using the observed mean of 2.0 worms per host for a dog in a single-dog household, we would expect a dog in a ten-dog household to have 1.26 worms (95\% Cl: 1.04-1.52).

Secondary analysis of the 2015-2018 dataset showed that the number of dogs per household was significantly and positively correlated with nearly all covariates, except for whether the dog was from a fishing village (Supplemental Table S3).

Univariable modeling for 2018 data also showed that dogs with a history of Guinea worm infection in a previous year were more likely to have higher worm burden (IRR $=1.16,95 \% \mathrm{CI}$ : 1.02-1.33, $P<0.05$ ) (Table 3). We found an inverse association between worm burden and the number of dogs present in the household (IRR $=0.95,95 \% \mathrm{Cl}: 0.91-0.98$, $P<0.005)$ and between worm burden and dog owners of the Sara Kaba ethnicity $(\mathrm{IRR}=0.86,95 \% \mathrm{Cl}$ : 0.77-0.98, $P<$ $0.05)$. In contrast to the results from the full dataset, dogs with high worm burden were less likely to come from a fishing village (IRR $=0.84,95 \% \mathrm{Cl}$ : 0.74-0.95, $P<0.005)$. Similar to the multivariable model for the full dataset (2015-2018), the final 2018 model showed that having a greater number of dogs in the household decreased the probability of high worm burden (AIRR $=0.94,95 \% \mathrm{Cl}$ : $0.91-0.98, P<0.005)$ after adjusting for dog age (AIRR = $0.98,95 \% \mathrm{Cl}: 0.94-1.02, P>0.1$ ). This translates to a $6 \%$ decrease in the number of worms per dog with each additional dog in a household.

Sensitivity analysis. Results for the truncated 2015-2018 data (that did not include the extreme observation of the dog with 79 worms) were similar to the results described earlier. Again, dogs with a Guinea worm infection in a previous year were more likely to have higher worm burden (IRR $=1.33,95 \%$ Cl: 1.20-1.47, $P<0.0001)$. Owner ethnicity (Sara Kaba) was negatively correlated with worm burden (IRR $=0.91,95 \% \mathrm{Cl}$ :

TABLE 1

Proportion of contained worms by emergence order

\begin{tabular}{|c|c|c|c|c|c|c|c|c|c|c|c|c|c|c|}
\hline \multirow[b]{4}{*}{$\begin{array}{l}\text { Worm } \\
\text { sequence }\end{array}$} & \multicolumn{14}{|c|}{ Proportion of contained worms } \\
\hline & \multirow{2}{*}{\multicolumn{2}{|c|}{$\begin{array}{l}\text { Total worms } \\
\text { contained }\end{array}$}} & \multicolumn{4}{|c|}{ Dogs with two worms } & \multicolumn{4}{|c|}{ Dogs with three worms } & \multicolumn{4}{|c|}{ Dogs with four worms } \\
\hline & & & \multicolumn{4}{|c|}{$(n=575)$} & \multicolumn{4}{|c|}{$(n=251)$} & \multicolumn{4}{|c|}{$(n=108)$} \\
\hline & $N$ & $\begin{array}{c}(\% \\
\text { contained) }\end{array}$ & $n$ & $\begin{array}{c}(\% \\
\text { contained) }\end{array}$ & $x^{2}$ & $P$-value & $n$ & $\begin{array}{c}(\% \\
\text { contained) }\end{array}$ & $x^{2}$ & $P$-value & $n$ & $\begin{array}{c}(\% \\
\text { contained) }\end{array}$ & $x^{2}$ & $\begin{array}{c}P- \\
\text { value }\end{array}$ \\
\hline Worm 1 & 677 & $(72.5)$ & 426 & $(74.1)$ & - & - & 181 & (72.1) & - & - & 70 & $(64.8)$ & - & - \\
\hline Worm 2 & 769 & (82.3) & 474 & (82.4) & - & - & 206 & (82.1) & - & - & 89 & (82.4) & - & - \\
\hline Worm 3 & 297 & (82.7) & NA & - & - & - & 205 & (81.7) & - & - & 92 & (85.2) & - & - \\
\hline \multirow[t]{2}{*}{ Worm 4} & 90 & (83.3) & NA & - & - & - & NA & - & - & - & 90 & (83.3) & - & - \\
\hline & - & - & - & - & 11.29 & $<0.001$ & - & - & 9.49 & $<0.01$ & - & - & 17.53 & $<0.001$ \\
\hline
\end{tabular}

Among dogs with multiple worms, worms that emerged later in the sequence were significantly more likely to be contained. Bold indicates statistical significance. 


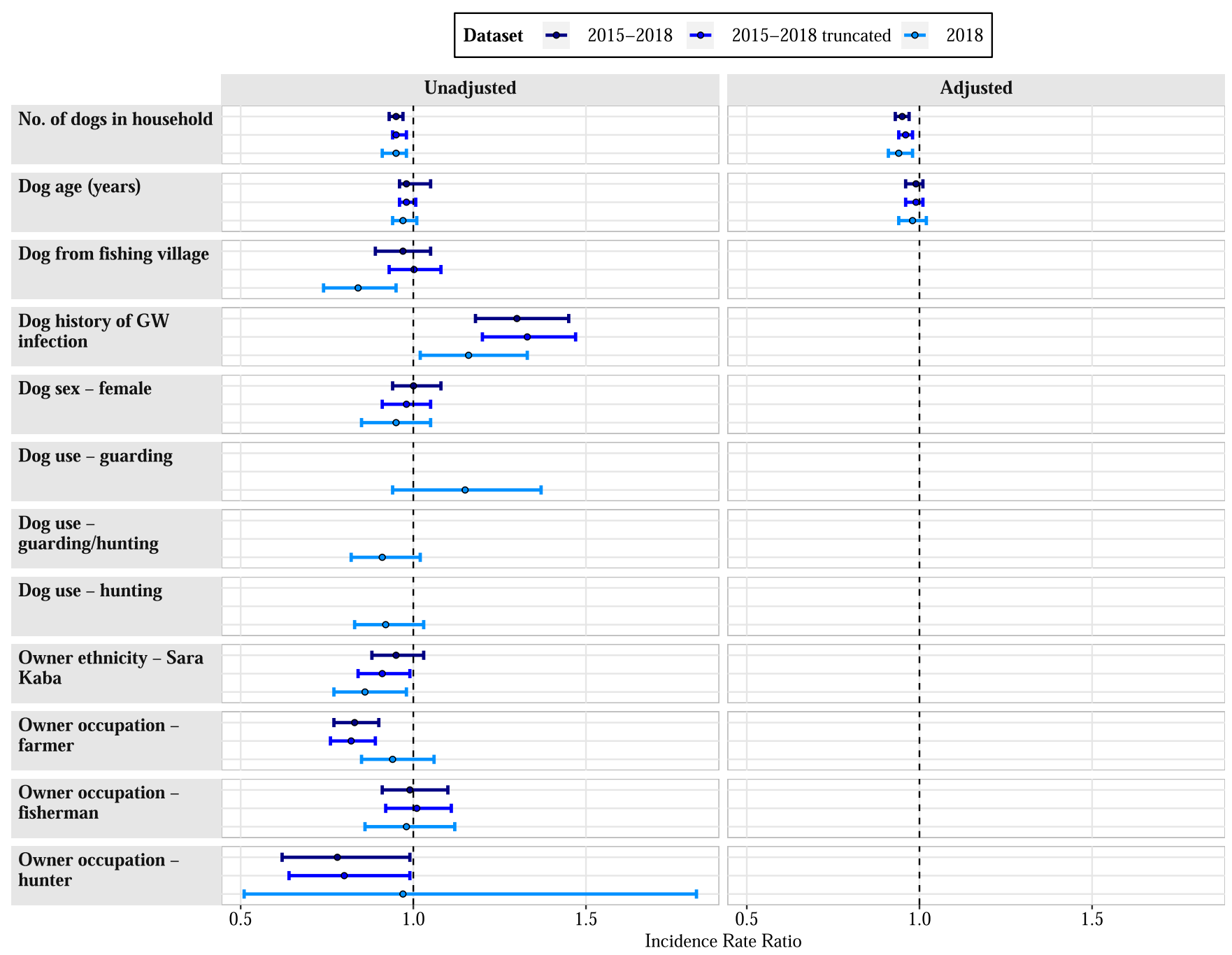

FIGURE 2. Comparison of negative binomial regression models. For all input datasets used, dogs with a history of previous Guinea worm infection in a different year were more likely to have elevated worm burden, and the number of dogs present in the household was negatively correlated with worm burden. Multivariable models were consistent for all input datasets. This figure appears in color at www.ajtmh.org.

0.84-0.99, $P<0.05)$, as were the owner occupations of farming (IRR $=0.82,95 \% \mathrm{Cl}: 0.76-0.89, P<0.0001)$ and hunting (IRR $=0.80,95 \% \mathrm{Cl}: 0.64-0.99, P<0.05$ ) (Supplemental Table S4). Multivariable modeling showed that the number of dogs in the household was negatively correlated with worm burden (AIRR $=0.96,95 \% \mathrm{Cl}$ : 0.94-0.98, $P<$ $0.0001)$, after adjusting for dog age (AIRR $=0.99,95 \% \mathrm{Cl}$ : 0.96-1.01, $P>0.1)$.

TABLE 2

Univariable and multivariable negative binomial regression models of worm burden, 2015-2018 data

\begin{tabular}{|c|c|c|c|c|c|c|}
\hline Variable (reference category) & Univariable, $n$ & $\begin{array}{l}\text { Incidence rate ratio } \\
\qquad(95 \% \mathrm{Cl})\end{array}$ & $P$-value & Multivariable, $n$ & $\begin{array}{l}\text { Adjusted incidence rate ratio } \\
\qquad(95 \% \mathrm{Cl})\end{array}$ & $P$-value \\
\hline No. of dogs in household & 3,285 & $0.95(0.93-0.97)$ & $<0.0001$ & 3,226 & $0.95(0.93-0.97)$ & $<0.0001$ \\
\hline Dog age (years) & 3,280 & $0.98(0.96-1.005)$ & $>0.1$ & - & $0.99(0.96-1.01)$ & $>0.1$ \\
\hline $\begin{array}{l}\text { Dog from fishing village (not from fishing } \\
\text { village) }\end{array}$ & 3,217 & $0.97(0.89-1.05)$ & $>0.1$ & - & - & - \\
\hline $\begin{array}{l}\text { Dog has history of GW infection in a } \\
\text { previous year }\end{array}$ & 3,338 & $1.30(1.18-1.45)$ & $<0.0001$ & - & - & - \\
\hline Dog sex, female (male) & 3,325 & $1.0005(0.94-1.08)$ & $>0.5$ & - & - & - \\
\hline $\begin{array}{l}\text { Owner ethnicity, Sara Kaba (other } \\
\text { ethnicity) }\end{array}$ & 3,345 & $0.95(0.88-1.03)$ & $>0.1$ & - & - & - \\
\hline Owner occupation, farmer (not a farmer) & 3,345 & $0.83(0.77-0.90)$ & $<0.0001$ & - & - & - \\
\hline $\begin{array}{l}\text { Owner occupation, fisherman (not a } \\
\text { fisherman) }\end{array}$ & 3,345 & $0.99(0.91-1.10)$ & $>0.5$ & - & - & - \\
\hline Owner occupation, hunter (not a hunter) & 3,345 & $0.78(0.62-0.99)$ & $<0.05$ & - & - & - \\
\hline
\end{tabular}


TABLE 3

Univariable and multivariable negative binomial regression models of worm burden, 2018 data

\begin{tabular}{|c|c|c|c|c|c|c|}
\hline Variable (reference category) & Univariable, $n$ & Incidence rate ratio $(95 \% \mathrm{Cl})$ & $P$-value & Multivariable, $n$ & $\begin{array}{l}\text { Adjusted incidence rate } \\
\text { ratio }(95 \% \mathrm{Cl})\end{array}$ & $P$-value \\
\hline No. of dogs in household & 1,004 & $0.95(0.91-0.98)$ & $<0.005$ & 983 & $0.94(0.91-0.98)$ & $<0.005$ \\
\hline Dog age (years) & 999 & $0.97(0.94-1.01)$ & $>0.1$ & - & $0.98(0.94-1.02)$ & $>0.1$ \\
\hline $\begin{array}{l}\text { Dog from fishing village (not from fishing } \\
\text { village) }\end{array}$ & 990 & $0.84(0.74-0.95)$ & $<0.005$ & - & - & - \\
\hline $\begin{array}{l}\text { Dog has history of GW infection in a } \\
\text { previous year }\end{array}$ & 1,020 & $1.16(1.02-1.33)$ & $<0.05$ & - & - & - \\
\hline Dog sex, female (male) & 1,012 & $0.95(0.85-1.05)$ & $>0.1$ & - & - & - \\
\hline Dog use, guarding (not used for guarding) & 1,018 & $1.15(0.94-1.37)$ & $>0.1$ & - & - & - \\
\hline $\begin{array}{l}\text { Dog use, guarding/hunting (not used for } \\
\text { guarding/hunting) }\end{array}$ & 1,020 & $0.91(0.82-1.02)$ & $>0.05$ & - & - & - \\
\hline Dog use, hunting (not used for hunting) & 1,014 & $0.92(0.83-1.03)$ & $>0.05$ & - & - & - \\
\hline $\begin{array}{l}\text { Owner ethnicity, Sara Kaba (other } \\
\text { ethnicity) }\end{array}$ & 1,020 & $0.86(0.77-0.98)$ & $<0.05$ & - & - & - \\
\hline Owner occupation, farmer (not a farmer) & 1,020 & $0.94(0.85-1.06)$ & $>0.1$ & - & - & - \\
\hline $\begin{array}{l}\text { Owner occupation, fisherman (not a } \\
\text { fisherman) }\end{array}$ & 1,020 & $0.98(0.86-1.12)$ & $>0.5$ & - & - & - \\
\hline Owner occupation, hunter (not a hunter) & 1,020 & $0.97(0.51-1.82)$ & $>0.5$ & - & - & - \\
\hline
\end{tabular}

An increased number of dogs in the household was negatively correlated with worm burden for both univariable and multivariable models (when adjusting for dog age). Univariable models showed that risk of elevated worm burden was greater for dogs with a history of Guinea worm infection; decreased risk of elevated worm burden was associated with owner ethnicity (Sara Kaba) and whether the dog came from a fishing village. Bold indicates statistical significance.

\section{DISCUSSION}

Data collected through active Guinea worm surveillance can help identify associations and broad trends in canine infections, steering eradication efforts more efficiently. Still, questions remain about clustering of Guinea worm at several scales, including the clustering of worms within dogs, infected dogs within households, and households with dog infections within villages. Results from this analysis offer insights about the first of these scenarios, the clustering of multiple worms within dogs.

Associations between household and owner characteristics suggest that anthropogenic characteristics are drivers of elevated worm burden in dogs. Our results showed that after adjusting for dog age, worm burden decreased as the number of dogs in the household increased, perhaps related to lesser amounts of any single contaminated food source available to an individual dog. Alternatively, low-burden canine cases may go undetected because more dogs in the household result in less attention paid to each individual animal. Other works have shown a positive correlation between group size and number of parasites per individual host, contrasting with our finding that group size decreases Guinea worm burden among infected dogs. ${ }^{20}$ This juxtaposition might relate to mobility of hosts and parasites, which confounds the relationship between group size and parasite intensity. ${ }^{20}$ In the Guinea worm context, dog mobility has been observed in Chad, ${ }^{17}$ and although the parasites themselves are not mobile, they are carried by other mobile hosts including humans and cats. ${ }^{2,3}$

Our secondary analysis showed that many of the covariates considered were positively correlated with the number of dogs in the household (among households with canine Guinea worm infection), suggesting that dog ownership is the product of a confluence of factors including owner occupation and ethnicity (Supplemental Table S3). CGWEP surveillance data, however, only collects information about infected dogs, and we therefore cannot broadly apply these findings to households with and without canine Guinea worm. Detailed surveys of randomly sampled households could further elucidate correlates of dog ownership and how this relates to the abundance of canine Guinea worm and owner knowledge and awareness of Guinea worm. Mbilo et al. ${ }^{21}$ showed that dog ownership was much more prevalent in Christian-oriented southern Chad in comparison with Muslim-oriented northern Chad, but did not consider ethnicity in their analysis. Most human and Guinea worm cases are concentrated in southern Chad ${ }^{4,16}$ so more detailed consideration of owner ethnicity and occupation within affected areas would be required.

A history of Guinea worm infection in a previous year was consistently associated with elevated worm burden across datasets. Although this variable was overshadowed by dog ownership in multivariable models, it is worthy of further consideration because of its programmatic utility. It is relatively easy for the field staff to use surveillance data identify dogs with a history of Guinea worm, in comparison with other variables considered such as the number of dogs per household, as dog ownership is likely to vary over time. From a practical standpoint, more vigilant surveillance should perhaps be applied to dogs with a history of Guinea worm because these animals are about 1.3 times more likely to have multiple worms, and because they may be more likely to experience recurrent infection, as has been demonstrated in humans. ${ }^{22} \mathrm{~A}$ previous analysis of surveillance data showed that dogs with recurrent infection represent approximately $13 \%$ of all infected dogs and that containment success has improved over time. ${ }^{16}$ Individual-level tracking of dogs with a history of infection, perhaps via micro-chipping, could further improve early detection and containment, ultimately reducing the number of water contamination events per adult worm.

Underlying observations from this analysis is the question: What causes elevated worm burden in some dogs, while others present with just a single worm? One explanation might be that greater infective dose results in more adult worms-but comparisons across historical studies are problematic because different inoculation methods are used, and many were not designed to explicitly test the dose-response relationship. Experiments involving ferret or dog infection with D. medinensis or its close relative Dracunculus insignis have shown a range of adult worms produced even when the infective dose is constant, ${ }^{23-26}$ indicating that infective dose 
alone is not the only driver of worm burden. Although initial infection requires ingestion of infectious copepods, additional factors hypothesized to influence worm burden include levels of gastric acid in the host stomach (which could impact larval survival ${ }^{25}$ ) and transmission route (because transport or paratenic hosts may serve as concentrators of infected copepods ${ }^{23}$ ). Regarding the latter, a field study did not show significant correlation between worm burden and consumption of fish-although the sample size was relatively small. ${ }^{17}$

Findings presented in this report are associative in nature and do not conclusively address the causes associated with elevated Guinea worm burden in dogs. It is impossible to tell from surveillance data alone whether dogs with multiple worms are the result of a single episode or of multiple sequential episodes of infection during the preceding year. To thoroughly explore this, more detailed exposure analysis would be required in conjunction with analysis of mitochondrial and microsatellite variation, ${ }^{27}$ which could be used to distinguish between maternal lineages. (However, genetic analyses would not identify repeat exposure to the same larval clutch, highlighting the need for integrated epidemiologic and genetic methods.) Many of the associations uncovered from this analysis are highly statistically significant but are weak (with IRRs and AIRRs close to 1), and practical implications therefore may be limited. Furthermore, occupation may not be well defined, as many owners have seasonal jobs and reported occupation may depend on the season in which they were interviewed. Not all worms were laboratory confirmed, and previous dog infections were reported by their owners and not necessarily by surveillance records. (A supplemental approach to identifying dogs with recurrent infections might be to search the surveillance data for dogs and owners that appear repeatedly in the multiyear dataset using fuzzy matching approaches.) The CGWEP does not track individual dogs over time, and therefore we are also unable to account for the impact of mortality between initial detection and the end of the sampling period. Relatedly, the surveillance system does not capture which dogs belong to the same households-and we therefore cannot control for the nonindependent effects of multiple dogs within a household in our models. Efforts are currently underway to use unique dog-level identifiers to aid in capturing accurate dog-level and household-level data.

Despite these potential limitations, these data represent the most comprehensive information worldwide about $D$. medinensis occurrence in dogs, and results from this analysis may help guide future eradication efforts. Dogs presenting with multiple adult Guinea worms is a common occurrence: of all infected dogs detected by the CGWEP surveillance system during the period of interest, approximately $40 \%$ had multiple worms. The rapid and effective containment of dogs with multi-worm infections has great potential to disrupt transmission, and therefore these animals warrant special attention. Further research is needed to elucidate relationships between humans and dogs in Chad, and to assess the efficacy of containment strategies and other interventions.

Received December 13, 2019. Accepted for publication December 24, 2020.

Published online February 22, 2021.

Note: Supplemental tables and figure appear at www.ajtmh.org.

Acknowledgments: We thank Mark Eberhard, Liz Thiele, and Fernando Torres-Velez for their comments on this manuscript before publication. We are also grateful to the CGWEP field staff for their contributions to data collection.

Disclaimer: The findings and conclusions in this report are those of the authors and do not necessarily represent the official position of the U.S. Department of Health and Human Services, the CDC, or the authors' affiliated institutions.

Authors' addresses: Sarah Anne J. Guagliardo, Ryan Wiegand, and Sharon L. Roy, Centers for Disease Control and Prevention, Atlanta, GA, E-mails: sguagliardo@cdc.gov, fwk2@cdc.gov, and str2@ cdc.gov. Christopher A. Cleveland, Southeastern Cooperative Wildlife Disease Study, University of Georgia, Athens, GA, E-mail: ccleve@ uga.edu. Hubert Zirimwabagabo and Elisabeth Chop, Guinea Worm Eradication Program, The Carter Center Chad Office, N'Djamena, Chad, E-mails: hubert.zirimwabagabo@cartercenter.org and echop711@ gmail.com. Philippe Tchindebet Ouakou, Guinea Worm Eradication Program, Ministry of Public Health, N'Djamena, Chad, E-mail: tchindebetouakou14@gmail.com. Ernesto Ruiz-Tiben, Donald R. Hopkins, and Adam J. Weiss, Guinea Worm Eradication Program, The Carter Center, Atlanta, GA, E-mails: eruizti@emory.edu, shandal.sullivan@cartercenter. org, and adam.weiss@cartercenter.org.

This is an open-access article distributed under the terms of the Creative Commons Attribution (CC-BY) License, which permits unrestricted use, distribution, and reproduction in any medium, provided the original author and source are credited.

\section{REFERENCES}

1. Watts SJ, 1987. Dracunculiasis in Africa in 1986: its geographic extent, incidence, and at-risk population. Am J Trop Med Hyg 37: $119-125$

2. Hopkins DR, Ruiz-Tiben E, Weiss AJ, Roy SL, Zingeser J, Guagliardo SAJ, 2018. Progress toward global eradication of dracunculiasis - January 2017-June 2018. MMWR Morb Mortal Wkly Rep 67: 1265-1270.

3. Hopkins DR, Weiss AJ, Roy SL, Zingeser J, Guagliardo SAJ, 2019. Progress toward global eradication of dracunculiasis - January 2018-June 2019. MMWR Morb Mortal Wkly Rep 68: 979-984.

4. Eberhard ML et al., 2014. The peculiar epidemiology of dracunculiasis in Chad. Am J Trop Med Hyg 90: 61-70.

5. Molyneux DH, Eberhard ML, Cleaveland S, Addey R, Guiguemde RT, Kumar A, Magnussen P, Breman JG, 2020. Certifying Guinea worm eradication: current challenges. Lancet 396: 1857-1860.

6. Lalitha CM, Anandan R, 1980. Guinea worm infection in dogs. Cheiron 9: 198-199.

7. Chun Sun F, 1958. Dracunculus infection in dogs in Kazakhstan. Med Parazitol (Mosk) 27: 219-220.

8. Chun Sun F, 1966. A case of Dracunculus infection in a domestic cat in the Kazakh SSR. Med Parazitol (Mosk) 35: 374-375.

9. Ghenis DE, 1972. New cases of Dracunculus medinensis L., 1758 detected in domestic cats and dogs in Kazakhstan. Med Parazitol (Mosk) 41: 365.

10. Litvinov VF, Vitvinov VP, 1981. Helminths of predatory mammals from easter Azerbaijan SSR, USSR. Parazitologiia 15: 219-223.

11. Velikanov BP, 1984. A case of Dracunculus medinensis infection in a dog in Turkmenia. Izvestiia Akademii Nauk Turkmenskoi SSR Seriia Biologicheskikh Nauk 1: 64-65.

12. The L.M Isaev Institute of Medical Parasitology, Uzebekistan Ministry of Health, 1998. Dracunculiasis Eradication in Uzebekistan: Country Report. Geneva, Switzerland: The World Health Organization.

13. Cairncross S, Muller R, Zagaria N, 2002. Dracunculiasis (Guinea worm disease) and the eradication initiative. Clin Microbiol Rev 15: $223-246$.

14. Muller R, 1971. Dracunculus and dracunculiasis. Adv Parasitol 9: 73-151.

15. Ruiz-Tiben E, Hopkins DR, 2006. Dracunculiasis (Guinea worm disease) eradication. Adv Parasitol 61: 275-309.

16. Guagliardo SAJ, Roy SL, Ruiz-Tiben E, Zirimwabagabo $\mathrm{H}$, Romero M, Chop E, Ouakou PT, Hopkins DR, Weiss AJ, 2020. Guinea worm in domestic dogs in Chad: a description and analysis of surveillance data. PLoS Negl Trop Dis 14: e0008207. 
17. McDonald RA, Wilson-Aggarwal JK, Swan GJF, Goodwin CD, Moundai T, Sankara D, Biswas G, Zingeser JA, 2020. Ecology of domestic dogs Canis familiaris as an emerging reservoir of Guinea worm Dracunculus medinensis infection. PLoS Negl Trop Dis 14: e0008170.

18. Wickham H, 2016. ggplot2: Elegant graphics for data analysis. New York, NY: Springer Verlag.

19. Tibshirani $R, 1996$. Regression shrinkage and selection via the lasso. J R Stat Soc 58: 267-288.

20. Patterson JEH, Ruckstuhl KE, 2013. Parasite infection and host group size: a meta-analytical review. Parasitology 140: 803-813.

21. Mbilo C, Léchenne M, Hattendorf J, Madjadinan S, Anyiam F, Zinsstag J, 2017. Rabies awareness and dog ownership among rural northern and southern Chadian communities-analysis of a community-based, cross-sectional household survey. Acta Trop 175: 100-110.
22. Tayeh A, Cairncross S, Maude GH, 1993. Water sources and other determinants of dracunculiasis in the northern region of Ghana. $J$ Helminthol 67: 213-225.

23. Cleveland CA, Eberhard ML, Thompson AT, Smith SJ, Zirimwabagabo $\mathrm{H}$, Bringolf R, Yabsley MJ, 2017. Possible role of fish as transport hosts for Dracunculus spp. lavae. Emerg Infect Dis 23: 1590-1592.

24. Onabamiro SD, 1956. The early stages of the development of Dracunculus medinensis (Linnaeus) in the mammalian host. Ann Trop Med Parasitol 50: 157-166.

25. Moorthy VN, Sweet WC, 1938. Further notes on the experimental infection of dogs with dracontiasis. Am J Epidemiol 27: 301-310.

26. Eberhard ML, Ruiz-Tiben E, Wallace SV, 1988. Dracunculus insignis: experimental infection in the ferret, Mustela putorius furo. J Helminthol 62: 265-270.

27. Thiele EA, Eberhard ML, Cotton JA, Durrant C, Berg J, Hamm K, RuizTiben E, 2018. Population genetic analysis of Chadian Guinea worms reveals that human and non-human hosts share common parasite populations. PLoS Negl Trop Dis 12: e0006747. 